\title{
Acúmulo de Nutrientes e Matéria Seca na Parte Aérea de Dois Cultivares de SoJa RR Sob Efeito de Formulações de GLYPHOSATE ${ }^{1}$
}

\author{
Nutrient and Shoot Dry Matter Accumulation of Two GR Soybean Cultivars under the Effect of \\ Glyphosate Formulations
}

\begin{abstract}
CAVALIERI, S.D. ${ }^{2}$, VELINI, E.D. ${ }^{3}$, SILVA, F.M.L. ${ }^{4}$, SÃO JOSÉ, A.R. ${ }^{5}$ e ANDRADE, G.J.M. ${ }^{6}$
RESUMO - O estudo da seletividade e dos efeitos secundários dos herbicidas nas culturas agrícolas é de extrema importância para o sucesso da agricultura. Objetivou-se com este trabalho avaliar o efeito de formulações de glyphosate sobre o acúmulo de nutrientes e produção de matéria seca na parte aérea de dois cultivares de soja resistente ao glyphosate (RR). O experimento foi conduzido em casa de vegetação, em delineamento de blocos ao acaso com seis repetições. Os tratamentos resultaram do arranjo fatorial entre formulações de glyphosate (Roundup Original ${ }^{\circledR}$, Roundup Ready ${ }^{\circledR}$, Roundup Transorb ${ }^{\circledR}$, Roundup WG ${ }^{\circledR}$, Roundup Ultra ${ }^{\circledR}$ e Zapp Qi ${ }^{\circledR}$ ), mais uma testemunha e cultivares de soja RR (CD 225 RR e $\mathrm{V}$ Max RR). As aplicações dos herbicidas ocorreram quando as plantas se apresentavam no estádio V3, na dosagem de 960 g e.a. ha-1. O acúmulo de macronutrientes e micronutrientes e a produção de matéria seca na parte aérea das plantas sempre foram maiores no cultivar $\mathrm{V}$ Max RR em relação ao CD 225 RR. As formulações Roundup Ready ${ }^{\circledR}$ e Roundup Ultra ${ }^{\circledR}$ não proporcionaram redução no acúmulo de nutrientes e na produção de matéria seca na parte aérea dos cultivares. Por sua vez, Roundup Transorb ${ }^{\circledR}$, Roundup Original ${ }^{\circledR}$ e Roundup WG ${ }^{\circledR}$ prejudicaram a nutrição dos cultivares e a produção de matéria seca. Conclui-se que o acúmulo de nutrientes e a produção de matéria seca na parte aérea de plantas de soja são alterados em função da aplicação de glyphosate, mesmo para cultivares RR.
\end{abstract}

Palavras-chave: Glycine max, herbicida, inibidores da EPSPs, nutrição, seletividade.

\begin{abstract}
The study of selectivity and secondary effects of herbicides on crops is extremely important to successful agriculture. This research aimed to evaluate the effect of glyphosate formulations on nutrient accumulation and dry matter production on the shoot of two glyphosateresistant (GR) soybean cultivars. The assay was carried out in a greenhouse and arranged in a randomized complete block design, replicated six times. The treatments were in a factorial arrangement including six glyphosate formulations (Roundup Original ${ }^{\circledR}$, Roundup Ready ${ }^{\circledR}$, Roundup Transorb ${ }^{\circledR}$, Roundup $W G^{\circledR}$, Roundup Ultra ${ }^{\circledR}$ and Zapp $\left.Q^{\circledR}\right)$, plus a control treatment, and two soybean cultivars (CD 225 RR and V Max RR). The herbicide applications were performed when the plants were at the $V 3$ growth stage, using a dose of $960 \mathrm{~g}$ a.e. $h \mathrm{a}^{-1}$. The macronutrient and micronutrient accumulation and dry matter production in the shoot of the soybean plants were greater in V Max RR cultivar than in CD 225 RR cultivar. The formulations Roundup Ready ${ }^{\circledR}$ and Roundup Ultra ${ }^{\circledR}$ did not promote nutrient accumulation reduction in the shoot of the cultivars. In addition, the formulations Roundup Original ${ }^{\circledR}$, Roundup Transor $b^{\circledR}$ and Roundup $W G^{\circledR}$ caused the greatest damage to nutrient accumulation and dry matter production. It was concluded that nutrient accumulation and dry matter production in the shoots of the soybean plants are affected by glyphosate application, even for GR cultivars.
\end{abstract}

Keywords: Glycine max, herbicide, EPSP inhibitors, nutrition, selectivity.

Recebido para publicação em 12.4.2011 e aprovado em 9.2.2012.

2 Engo-Agrº., D.Sc., Pesquisador da Embrapa Hortaliças, Rod. BR 060, Km 9, Brasília/Anápolis, $70359-970$ Brasília-DF, <cavalieri@cnph.embrapa.br>; ${ }^{3}$ Engo-Agro ., D.Sc., Professor da Faculdade de Ciências Agronômicas, Universidade Estadual Paulista "Júlio de Mesquita Filho", Núcleo de Pesquisas Avançadas em Matologia - FCA/UNESP/NUPAM, $18603-970$ Botucatu-SP;

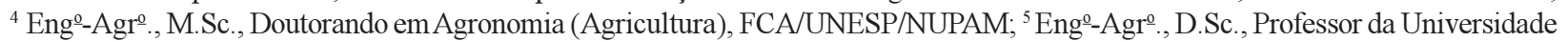
Estadual do Sudoeste da Bahia - UESB, Dep. de Fitotecnia e Zootecnia, Estrada do Bem Querer, Km 4, $45083-900$ Vitória da Conquista-BA; ${ }^{6}$ Eng - -Agr ${ }^{\circ}$., M.Sc., Representante Comercial, ANDRADE AGRI Representação Comercial LTDA, Rua Agenor Gazonato, nำ176, 18500-000 Laranjal Paulista-SP.

Planta Daninha, Viçosa-MG, v. 30, n. 2, p. 349-358, 2012 


\section{INTRODUÇÃO}

A soja (Glycine max) é uma das culturas agrícolas mais importantes do Brasil, com área cultivada de 24,97 milhões de hectares e produção de 68,75 milhões de toneladas na safra 2011/2012, com produtividade média de $2.753 \mathrm{~kg} \mathrm{ha}^{-1}$ (CONAB, 2012). No ano de 2011, a área cultivada com sementes transgênicas no mundo foi de 160 milhões de hectares. Desse total, o Brasil cultivou 30,3 milhões de hectares com culturas geneticamente modificadas, ocupando o segundo lugar no ranking mundial de transgênicos, ficando atrás apenas dos Estados Unidos (69 milhões de hectares) (ISAAA, 2011). Estima-se que 21,4 milhões de hectares ou $85,3 \%$ da área seriam cultivados com cultivares transgênicas na safra 2011/ 2012, aumento de $16,7 \%$ em relação à safra anterior, segundo Céleres (2011).

O glyphosate é um herbicida não seletivo, de amplo espectro, aplicado em pós-emergência, que controla a maioria das espécies infestantes. Esse herbicida inibe a sintese de aminoácidos aromáticos (fenilalanina, tirosina e triptofano) em plantas e microrganismos que possuem o ciclo do ácido chiquímico atuando na enzima precursora EPSPs (5-enolpiruvilchiquimato-3-fosfato sintase), evitando a transformação do chiquimato em corismato (Shaner \& Bridges, 2003). No caso da soja RR, a tolerância ao herbicida foi obtida pela inserção de um gene (AroA) oriundo do genoma de Agrobacterium sp., estirpe CP4, o qual codifica uma variante da EPSPs (CP4 EPSPS), especialmente tolerante à inibição pelo glyphosate (Padgette et al., 1995). Sob tratamento com esse herbicida, as plantas de soja não são afetadas, em virtude da ação continuada e sistemática dessa enzima alternativa, insensivel ao produto.

No entanto, com o aumento do uso da tecnologia da soja RR, agricultores têm notado que alguns cultivares apresentam injúrias visuais logo após a aplicação do glyphosate (Santos et al., 2007a; Zablotowicz \& Reddy, 2007). Esse efeito pode estar relacionado ao aumento demasiado da dosagem aplicada; à aplicação de diferentes formulações de glyphosate, que incluem: maior intoxicação a organismos não alvo (Santos et al., 2005, 2006), maior velocidade de absorção e translocação (Molin
$\&$ Hirase, 2004) e desbalanço nutricional das plantas (Zobiole et al., 2009; Serra et al., 2011); à sensibilidade dos cultivares ao herbicida (Oliveira Jr et al., 2008); e/ou ao efeito de surfatantes. Entretanto, Reddy et al. (2004) atribuem os sintomas ao acúmulo do ácido aminometilfosfônico (AMPA) nos tecidos vegetais.

Há relatos de deficiência de $\mathrm{Fe}, \mathrm{Zn}$ e $\mathrm{Mn}$ na soja RR após a utilização frequente de glyphosate (Johal \& Huber, 2009). O sintoma típico observado após a aplicação do herbicida é o amarelecimento das folhas superiores. Recentes pesquisas demonstraram que esse sintoma clorótico nas culturas RR pode ser atribuído à imobilização de cátions bivalentes, como Fe e Mn (Bott et al., 2008; Zobiole et al., 2010a). Uma possibilidade para ocorrer menor disponibilidade desses cátions é que o glyphosate, por ser um ácido fosfônico e quelador de cátions metálicos (Coutinho \& Mazo, 2005), poderia formar quelatos com cátions bivalentes e trivalentes. Provavelmente, a duração desse amarelecimento depende da habilidade da planta de se recuperar pela absorção radicular dos elementos que foram imobilizados pelo glyphosate.

A introdução do gene de resistência ao glyphosate trouxe consequências negativas aos microrganismos simbióticos associados com a soja RR, devido à sua translocação para os nódulos radiculares (Reddy \& Zablotowicz, 2003) e exsudação do herbicida para a rizosfera das plantas (Kremer et al., 2005). As bactérias fixadoras de $\mathrm{N}_{2}$ da soja possuem a forma sensivel da enzima EPSPs e acumulam os ácidos chiquímico, hidroxibenzoico e protocatecuico quando expostas ao glyphosate, os quais, em altas concentrações, inibem o crescimento e induzem os nódulos radiculares à morte (De Maria et al., 2006). O efeito tóxico do glyphosate às bactérias também é atribuído à incapacidade dos organismos de sintetizarem aminoácidos aromáticos.

Levando em consideração que atualmente estão disponiveis no mercado diversas formulações de glyphosate - todas com o mesmo mecanismo de ação, independentemente dos sais utilizados - a soja RR pode sofrer injúrias em decorrência da aplicação dessas formulações, devido à presença de surfatantes ou do tipo de sal encontrado nelas (Reddy \& Zablotowicz, 2003). Os surfatantes, para promoverem maior 
intoxicação visual, são requeridos em concentrações maiores que as necessárias para reduzir a tensão superficial, o que indica que o modo de ação não está só limitado à sua ação como espalhante das gotas, mas também no aumento da permeabilidade da cutícula ou da membrana celular, ou de ambas.

No Brasil, a formulação Roundup Ready ${ }^{\circledR}$ é a única à base de glyphosate registrada para aplicação em soja RR. Entretanto, mesmo não possuindo registro, todas as outras formulações vêm sendo utilizadas. Assim, o estudo de efeitos secundários proporcionados pela aplicação de glyphosate em soja RR torna-se de grande importância, considerando a existência de diversas formulações e cultivares de soja RR no mercado. Dessa forma, objetivou-se com este trabalho avaliar o efeito de formulações comerciais de glyphosate sobre o acúmulo de nutrientes e produção de matéria seca da parte aérea de dois cultivares de soja RR.

\section{MATERIAL E MÉTODOS}

O presente estudo foi conduzido em maio de 2009, em casa de vegetação do Núcleo de Pesquisas Avançadas em Matologia (NUPAM), pertencente ao Departamento de Produção Vegetal da Faculdade de Ciências Agronômicas, UNESP, campus de Botucatu-SP.

As unidades experimentais foram constituídas por vasos de $7 \mathrm{~L}$ de capacidade volumétrica, usando como substrato amostras de Latossolo Vermelho distrófico (LVd), previamente corrigido e adubado após peneiramento (10 mesh), conforme análise química.

De acordo com as análises granulométrica e química, o solo usado no experimento apresentava textura média, com $20 \%$ de argila, $4 \%$ de silte e $76 \%$ de areia; $\mathrm{pH} \mathrm{CaCl} \mathrm{Cl}_{2}=$ 4,$2 ; \mathrm{H}^{+}+\mathrm{Al}^{3+}, \mathrm{Ca}^{2+}, \mathrm{Mg}^{2+}$ e K${ }^{+}$iguais a 72,$0 ; 3,0$; 1,0 ; e $0,5 \mathrm{mmol}_{\mathrm{c}} \mathrm{dm}^{-3}$, respectivamente; $\mathrm{B}, \mathrm{Cu}$, $\mathrm{Fe}, \mathrm{Mn}$ e $\mathrm{Zn}$ iguais a 0,$35 ; 0,6 ; 62,0 ; 0,5$; e $0,2 \mathrm{mg} \mathrm{dm}^{-3}$, respectivamente; $\mathrm{P}=2,0 \mathrm{mg} \mathrm{dm}^{-3}$; e $\mathrm{MO}=26 \mathrm{~g} \mathrm{dm}^{-3}$.

Os tratamentos obedeceram a um arranjo fatorial $7 \times 2$ entre seis formulações comerciais de glyphosate (Roundup Original ${ }^{\circledR}$, Roundup Ready $^{\circledR}$, Roundup Transorb ${ }^{\circledR}$, Roundup $\mathrm{WG}^{\circledR}$, Roundup Ultra ${ }^{\circledR}$ e Zapp $\mathrm{Qi}^{\circledR}$ ), mais uma testemunha e dois cultivares de soja RR
(CD 225 RR e V Max RR), ambos de grupo de maturação precoce. O delineamento experimental utilizado foi o de blocos ao acaso com seis repetições.

Antes da semeadura dos cultivares de soja, as sementes foram inoculadas com uma formulação comercial líquida, na concentração de $3 \times 10^{9}$ rizóbios por grama. Quando as plantas atingiram o estádio V1, foi feito desbaste, mantendo-se duas plantas por vaso. Durante a condução do ensaio, o solo dos vasos foi mantido com umidade próxima à da capacidade de campo.

As aplicações das formulações comerciais dos herbicidas ocorreram quando as plantas de soja apresentavam-se no estádio V3 (25 dias após a emergência). Para isso, a dosagem de glyphosate aplicada foi de 960 g e.a. ha ${ }^{-1}$, e utilizou-se de água deionizada para o preparo das caldas de pulverização.

As pulverizações com glyphosate foram feitas com auxílio de um equipamento instalado em laboratório. A barra de pulverização era constituída por quatro pontas XR 11002 VS, espaçadas de $0,5 \mathrm{~m}$ e posicionadas a $0,5 \mathrm{~m}$ de altura em relação à superficie dos alvos. Por ocasião da aplicação do herbicida, o sistema foi operado com velocidade de deslocamento de $3,6 \mathrm{~km} \mathrm{~h}^{-1}$ e volume de aplicação correspondente a $200 \mathrm{~L} \mathrm{ha}^{-1}$. O equipamento foi operado com pressão constante de $200 \mathrm{kPa}$, pressurizado por ar comprimido.

Após o término das aplicações, as caldas remanescentes foram levadas imediatamente para laboratório e analisadas quanto à concentração de macronutrientes $(\mathrm{N}, \mathrm{P}, \mathrm{K}, \mathrm{Ca}, \mathrm{Mg}$ e $\mathrm{S})$ e micronutrientes ( $\mathrm{Cu}, \mathrm{Fe}, \mathrm{Mn}, \mathrm{Zn}$ e B), com a finalidade de averiguar possiveis diferenças na concentração de nutrientes, as quais poderiam estar relacionadas com o maior ou menor acúmulo desses pelas plantas de soja.

Transcorridos 15 dias da aplicação dos tratamentos, a parte aérea das plantas de soja foi colhida, separada em folhas e hastes e secas em estufa de circulação forçada e renovação de ar por 72 horas, a uma temperatura de $65^{\circ} \mathrm{C}$. Depois de secas, obtiveram-se os dados de massa de matéria seca de folhas e hastes, além da massa de matéria seca de parte aérea (hastes + folhas); posteriormente, 
o material foi triturado e enviado para análise dos teores de macronutrientes e micronutrientes. Após obtenção dos resultados, procedeu-se aos cálculos referentes ao acúmulo de nutrientes na parte aérea das plantas em cada unidade experimental, para fins de comparação entre os tratamentos. Os dados foram submetidos à análise de variância, e suas médias, comparadas pelo teste $t$ $(\mathrm{p}<0,05)$.

\section{RESULTADOS E DISCUSSÃO}

De modo geral, considerando os cultivares de soja RR avaliados, verificou-se que o acúmulo de macronutrientes e micronutrientes na parte aérea das plantas sempre foi maior no cultivar V Max RR do que no CD 225 RR (Tabela 1), quando houve diferenças estatisticamente significativas entre eles. Esse fato evidencia maior sensibilidade do cultivar CD 225 RR em relação ao V Max RR quando submetidos às aplicações das formulações comerciais de glyphosate testadas. Entretanto, a superioridade do cultivar V Max RR em relação ao CD 225 RR persistiu para o acúmulo dos macronutrientes $\mathrm{N}$ e $\mathrm{K}$ e dos micronutrientes $\mathrm{Cu}$ e $\mathrm{Fe}$ mesmo no tratamento testemunha. Isso sugere maior habilidade do cultivar V Max RR em absorver esses nutrientes do solo - característica essa que pode ser atribuída a efeitos fisiológicos e ambientais ou ao próprio cultivar.

Diante do exposto, para os macronutrientes (Tabela 1) e levando-se em conta o fator cultivares, o acúmulo de $\mathrm{N}$ diferiu em todos os tratamentos, exceto para a formulação Roundup Ready ${ }^{\circledR}$, a qual possivelmente apresentou-se mais seletiva para o cultivar CD 225 RR, uma vez que o acúmulo desse elemento não foi afetado por ele. Em relação ao $\mathrm{P}$, tanto na testemunha sem herbicida quanto nos tratamentos, não se observou diferença significativa para o acúmulo do nutriente nos tecidos vegetais entre os cultivares. No que diz respeito ao K, não houve diferença significativa entre os cultivares somente para a formulação Roundup $W^{\circledR}{ }^{\circledR}$. Nesse caso, esse fato pode ser explicado pelo efeito negativo proporcionado ao cultivar V Max RR, que apresentou menor acúmulo de $\mathrm{K}$ em comparação com a respectiva testemunha.
Todos os tratamentos com as formulações de glyphosate reduziram o acúmulo de Ca no cultivar CD $225 \mathrm{RR}$, exceto a formulação Roundup $\operatorname{Ready}^{\circledR}$, a qual se destacou novamente como a mais seletiva. No que se refere ao $\mathrm{Mg}$, as formulações Roundup Original $^{\circledR}$ e Roundup Transorb ${ }^{\circledR}$ foram as que reduziram o acúmulo desse nutriente no cultivar CD $225 \mathrm{RR}$, em relação ao V Max RR. Já para o S, apenas o Roundup Origina ${ }^{\circledR}$ reduziu o acúmulo desse nutriente, em comparação com o cultivar V Max RR.

Esses resultados indicam que existe variabilidade entre os cultivares quanto à aquisição de nutrientes do solo, podendo o acúmulo desses pela parte aérea das plantas ser influenciado em menor ou maior intensidade pela aplicação das diferentes formulações de glyphosate. Nesse caso, vale a pena ressaltar que, independentemente da aplicação das formulações, houve maior acúmulo de $\mathrm{N}$ e $\mathrm{K}$ pela testemunha do cultivar V Max RR, evidenciando maior vigor desse em relação ao cultivar CD 225 RR. Por outro lado, o CD 225 RR demonstrou ser mais sensivel que o cultivar V Max RR quanto ao acúmulo de $\mathrm{Ca}, \mathrm{Mg}$ e $\mathrm{S}$.

Para o fator formulações de glyphosate, o acúmulo de $\mathrm{N}$ no cultivar CD $225 \mathrm{RR}$ foi afetado negativamente quando se aplicaram as formulações Roundup Transorb ${ }^{\circledR}$ e Roundup Original $^{\circledR}$, em relação à testemunha sem aplicação. No entanto, para o cultivar V Max RR, o acúmulo de $\mathrm{N}$ foi afetado negativamente somente pelo Roundup Transorb ${ }^{\circledR}$. Os herbicidas podem influenciar o metabolismo de $\mathrm{N}$ por meio de efeitos diretos nos rizóbios simbiontes (Zobiole et al., 2007; Santos et al., 2007b), reduzindo a nodulação nas plantas, ou indiretos na fisiologia do hospedeiro dessas, incapacitando os organismos de sintetizar aminoácidos aromáticos, os quais possuem a forma sensivel da enzima EPSPs. Zobiole et al. (2010b) relataram que o glyphosate pode afetar a fixação biológica do $\mathrm{N}$ devido à redução do teor de níquel, sendo este essencial para os microrganismos simbióticos. Kremer \& Means (2009) observaram desbalanço de ácido indol acético (AIA) com a aplicação de glyphosate em soja RR, o que levou à menor nodulação das raizes pelo Bradyrhizobium japonicum.

Com relação ao acúmulo de $\mathrm{P}$, para o cultivar CD 225 RR, somente a formulação 
Tabela 1 - Acúmulo de macronutrientes (N, P, K, Ca, Mg e S) e micronutrientes ( $\mathrm{Cu}, \mathrm{Fe}, \mathrm{Mn}, \mathrm{Zn}$ e B) pela parte aérea de cultivares de soja RR aos 15 dias após o tratamento (DAT) com formulações do herbicida glyphosate ( $960 \mathrm{~g} \mathrm{e.a} \mathrm{ha-1)}$

\begin{tabular}{|c|c|c|c|c|c|c|c|c|c|c|c|c|c|}
\hline \multirow{3}{*}{ Tratamento } & \multicolumn{13}{|c|}{ Acúmulo de macronutrientes (mg por planta) } \\
\hline & \multicolumn{2}{|l|}{$\mathrm{N}$} & \multicolumn{3}{|c|}{$\mathrm{P}$} & \multicolumn{2}{|r|}{ K } & \multicolumn{2}{|c|}{$\mathrm{Ca}$} & \multicolumn{2}{|c|}{$\mathrm{Mg}$} & \multicolumn{2}{|r|}{$\mathrm{S}$} \\
\hline & $\begin{array}{c}\text { CD 225 } \\
\text { RR }\end{array}$ & $\begin{array}{c}\text { V Max } \\
\text { RR }\end{array}$ & \multicolumn{2}{|c|}{$\begin{array}{c}\text { CD 225 } \\
\text { RR }\end{array}$} & $\begin{array}{c}\text { V Max } \\
\text { RR }\end{array}$ & $\begin{array}{c}\text { CD 225 } \\
\text { RR }\end{array}$ & $\begin{array}{l}\text { V Max } \\
\text { RR }\end{array}$ & $\begin{array}{c}\text { CD 225 } \\
\text { RR }\end{array}$ & $\begin{array}{c}\text { V Max } \\
\text { RR }\end{array}$ & \begin{tabular}{|c|} 
CD 225 \\
RR
\end{tabular} & $\begin{array}{c}\text { V Max } \\
\text { RR }\end{array}$ & $\begin{array}{c}\text { CD 225 } \\
\text { RR }\end{array}$ & $\begin{array}{c}\text { V Max } \\
\text { RR }\end{array}$ \\
\hline Testemunha & $26,2 a^{*}$ & $36,2 \mathrm{ab}$ & \multicolumn{2}{|c|}{$5,4 \mathrm{a}$} & $5,7 \mathrm{a}$ & $16,1 \mathrm{ab}^{*}$ & * $18,4 \mathrm{a}$ & $20,8 \mathrm{a}$ & $23,5 \mathrm{ab}$ & $9,6 \mathrm{a}$ & $9,2 \mathrm{a}$ & $2,7 \mathrm{ab}$ & $2,6 \mathrm{ab}$ \\
\hline Roundup Original $^{\mathbb{R}}$ & $19,2 b^{*}$ & \multirow{2}{*}{ 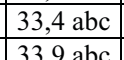 } & \multicolumn{2}{|c|}{$3,7 \mathrm{~b}$} & $4,7 \mathrm{a}$ & $11,6 b^{*}$ & $25,8 \mathrm{ab}$ & $14,4 \mathrm{bc} *$ & $21,5 \mathrm{~b}$ & $6,9 \mathrm{bc}^{*}$ & $9,3 \mathrm{a}$ & $1,9 \mathrm{~b}^{*}$ & $2,9 \mathrm{ab}$ \\
\hline Roundup Ready ${ }^{\mathbb{R}}$ & $29,1 \mathrm{a}$ & & \multicolumn{2}{|c|}{$5,7 \mathrm{a}$} & $5,8 \mathrm{a}$ & $18,4 \mathrm{a}^{*}$ & $28,3 \mathrm{a}$ & \begin{tabular}{|l|}
$20,0 \mathrm{a}$ \\
\end{tabular} & $23,6 \mathrm{ab}$ & $9,8 \mathrm{a}$ & $9,9 a$ & $3,1 \mathrm{a}$ & $3,4 \mathrm{a}$ \\
\hline RoundupTransorb $^{\circledR}$ & $20,4 b^{*}$ & $\begin{array}{l}33,9 a b c \\
30,3 c\end{array}$ & & $2 \mathrm{ab}$ & 5,7 & $11,5 b^{*}$ & $47,4 \mathrm{ab}$ & $13,5 \mathrm{c} *$ & $26,2 \mathrm{a}$ & $6 \mathrm{c}^{*}$ & $10,1 \mathrm{a}$ & $2,2 \mathrm{~b}$ & $2,6 \mathrm{ab}$ \\
\hline Roundup Ultra $^{\circledR}$ & $26,5 \mathrm{a}^{*}$ & $36,7 \mathrm{a}$ & 5, & $3 \mathrm{a}$ & 4,9 & $18,8 \mathrm{a}^{*}$ & $25,8 \mathrm{ab}$ & $19,2 a^{*}$ & $24,0 \mathrm{ab}$ & $5 \mathrm{a}$ & $9,0 a$ & $2,8 \mathrm{a}$ & $3,0 \mathrm{ab}$ \\
\hline Roundup $\mathrm{WG}^{(\mathbb{R}}$ & \begin{tabular}{|l|l}
$24,5 \mathrm{ab}^{*}$ & 3 \\
\end{tabular} & $31,0 \mathrm{bc}$ & 5, & $7 \mathrm{a}$ & 5,1 & $16,5 \mathrm{ab}$ & $21,3 \mathrm{~b}$ & $18,4 a^{*}$ & $23,1 \mathrm{ab}$ &, $0 \mathrm{a}$ & $9,0 \mathrm{a}$ & $2,6 \mathrm{ab}$ & $2,4 \mathrm{~b}$ \\
\hline Zapp Qi ${ }^{(R)}$ & $24,4 \mathrm{ab}^{*}$ & $36,1 \mathrm{ab}$ & & $8 \mathrm{ab}$ & 5,0 & $16,3 \mathrm{ab}^{*}$ & * $25,2 \mathrm{ab}$ & $18,0 \mathrm{ab}^{*}$ & $26,0 \mathrm{a}$ & $2 \mathrm{ab}$ & 9,8 a & $2,3 \mathrm{ab}$ & $3,0 \mathrm{ab}$ \\
\hline DMS & 5,6 & & & 1 & & & 5,1 & 4,0 & & 1, & & & 0,8 \\
\hline $\mathrm{CV}(\%)$ & 16,7 & & & 27 & & & 1,38 & 16,6 & & 15 , & & & 5,45 \\
\hline & & & & & & Acúmulo d & de micronut & ientes $(\mathrm{mg}$ & por planta) & & & & \\
\hline Tratamento & & $\mathrm{Cu}$ & & & & & & In & & $\mathrm{Zn}$ & & B & \\
\hline & $\begin{array}{c}\text { CD 225 } \\
\text { RR }\end{array}$ & $\begin{array}{l}\mathrm{V} \mathrm{Mc} \\
\mathrm{RR}\end{array}$ & & & $\begin{array}{l}225 \\
\mathrm{R}\end{array}$ & $\begin{array}{c}\text { V Max } \\
\text { RR }\end{array}$ & $\begin{array}{c}\text { CD 225 } \\
\text { RR }\end{array}$ & $\begin{array}{c}\mathrm{V} \text { Max } \\
\text { RR }\end{array}$ & $\begin{array}{c}\text { CD 225 } \\
\text { RR }\end{array}$ & $\begin{array}{r}\mathrm{VI} \\
\mathrm{R}\end{array}$ & & $\begin{array}{c}\text { CD 225 } \\
\text { RR }\end{array}$ & $\begin{array}{c}\text { V Max } \\
\text { RR }\end{array}$ \\
\hline Testemunha & $0,0033 \mathrm{~cd}^{*}$ & 0,0088 & & 0,43 & ab* & $0,57 \mathrm{abc}$ & $0,11 \mathrm{ab}$ & $0,16 \mathrm{~b}$ & $0,023 \mathrm{a}$ & 0,02 & $4 \mathrm{ab}$ & $0,09 \mathrm{ab}$ & $0,08 \mathrm{~b}$ \\
\hline Roundup Original $^{\mathbb{R}}$ & $0,0027 \mathrm{~d}^{*}$ & 0,0070 & & 0,32 & $a b^{*}$ & $0,53 \mathrm{bc}$ & $0,09 \mathrm{~b}^{*}$ & $0,17 \mathrm{ab}$ & $0,015 \mathrm{abc}$ & 0,02 & & $0,07 \mathrm{c}^{*}$ & $0,09 \mathrm{ab}$ \\
\hline Roundup Ready ${ }^{\mathbb{R}}$ & $0,0072 \mathrm{ab}$ & 0,0077 & & 0,45 & & $0,65 \mathrm{ab}$ & $0,14 \mathrm{ab}$ & $0,18 \mathrm{ab}$ & $0,022 \mathrm{ab}$ & 0,02 & $4 \mathrm{ab}$ & $0,10 \mathrm{a}$ & $0,10 \mathrm{a}$ \\
\hline RoundupTransorb ${ }^{\circledR}$ & $0,0052 \mathrm{bc}$ & 0,0062 & & 0,30 & & $0,65 \mathrm{ab}$ & $0,10 \mathrm{~b}^{*}$ & $0,17 \mathrm{~b}$ & $0,012 \mathrm{c}$ & 0,01 & & $0,07 \mathrm{c}^{*}$ & $0,08 \mathrm{ab}$ \\
\hline Roundup Ultra ${ }^{\circledR}$ & $0,0069 \mathrm{ab}$ & 0,0070 & & 0,33 & $a b^{*}$ & $0,66 \mathrm{ab}$ & $0,16 \mathrm{a}$ & $0,21 \mathrm{ab}$ & $0,019 a b c$ & 0,02 & & $0,09 \mathrm{ab}$ & $0,10 \mathrm{ab}$ \\
\hline Roundup $\mathrm{WG}^{(\mathbb{R})}$ & $0,0086 \mathrm{a}^{*}$ & 0,0062 & & 0,35 & & $0,48 \mathrm{c}$ & $0,14 \mathrm{ab}$ & $0,16 \mathrm{~b}$ & $0,017 \mathrm{abc}$ & 0,01 & & $0,08 \mathrm{bc}$ & $0,09 \mathrm{ab}$ \\
\hline Zapp Qi ${ }^{(\mathbb{R}}$ & $0,0060 \mathrm{~b}$ & 0,0075 & & 0,34 & $a b^{*}$ & $0,67 \mathrm{a}$ & $0,14 \mathrm{ab}^{*}$ & $0,22 \mathrm{a}$ & $0,013 b^{*}$ & 0,03 & & $0,08 \mathrm{bc}^{*}$ & $0,10 \mathrm{a}$ \\
\hline DMS & & 0,0023 & & & & 14 & & 05 & & 0,010 & & 0 , & 02 \\
\hline CV $(\%)$ & & 1,17 & & & & & &, 79 & & 1,44 & & 17 , & 66 \\
\hline
\end{tabular}

* = médias na linha diferem significativamente pelo teste $\mathrm{F}$ para a variável cultivar $(\mathrm{p}<0,05)$; Médias seguidas pela mesma letra na coluna não diferem significativamente pelo teste $\mathrm{t}(\mathrm{p}<0,05)$.

Roundup Original ${ }^{\circledR}$ reduziu o seu acúmulo, porém não foram constatadas diferenças significativas entre os tratamentos para o acúmulo desse macronutriente no cultivar $\mathrm{V}$ Max RR. No que diz respeito ao $\mathrm{K}$ e $\mathrm{S}$, no cultivar CD 225 RR o acúmulo desses nutrientes na testemunha foi semelhante ao encontrado nas plantas tratadas com as diferentes formulações de glyphosate. No entanto, o acúmulo de $\mathrm{K} \mathrm{e} \mathrm{S}$ observado nas plantas tratadas com Roundup Ready $^{\circledR}$ e Roundup Ultra ${ }^{\circledR}$ apresentou-se superior ao das formulações Roundup Transorb ${ }^{\circledR} \mathbf{e}$ Roundup Original ${ }^{\circledR}$, o que demonstra a segurança de utilização das primeiras em relação às últimas formulações. Para o cultivar V Max RR, a testemunha e o tratamento com Roundup Ready ${ }^{\circledR}$ foram significativamente superiores ao Roundup $\mathrm{WG}^{\circledR}$, quanto ao acúmulo de K. Para esse cultivar, o Roundup Ready ${ }^{\circledR}$ também apresentou acúmulo de $\mathrm{S}$ superior ao do Roundup WG ${ }^{\circledR}$.

Com relação ao $\mathrm{Ca}$ e $\mathrm{Mg}$, no cultivar CD 225 RR, os dados de acúmulo encontrados nos tratamentos testemunha, Roundup Ready $^{\circledR}$, Roundup Ultra ${ }^{\circledR}$ e Roundup WG ${ }^{\circledR}$ foram semelhantes entre si e superiores aos da aplicação de Roundup Original ${ }^{\circledR}$ e Roundup Transorb ${ }^{\circledR}$. Para o cultivar V Max RR, o acúmulo de Mg não diferiu entre os tratamentos. Entretanto, para o acúmulo de $\mathrm{Ca}$, a formulação Roundup Original ${ }^{\circledR}$ apresentou-se significativamente inferior às formulações Roundup Transorb $^{\circledR}$ e Zapp Qi ${ }^{\circledR}$, e todos os tratamentos não diferiram da testemunha.

Quanto ao acúmulo de micronutrientes no fator cultivares (Tabela 1), o CD $225 \mathrm{RR}$ apresentou acúmulo de $\mathrm{Cu}$ significativamente inferior ao do V Max RR para os tratamentos testemunha, Roundup Original ${ }^{\circledR}$ e Roundup $\mathrm{WG}^{\circledR}$. Contudo, para o Fe, com exceção do Roundup $\mathrm{WG}^{\circledR}$, todos os tratamentos promoveram redução do acúmulo no cultivar CD 225 RR em relação ao V Max RR. Já para o Mn e B, o cultivar CD 225 RR somente apresentou menor acúmulo para as formulações Roundup Original $^{\circledR}$, Roundup Transorb ${ }^{\circledR}$ e 
Zapp Qi ${ }^{\circledR}$. No entanto, para o $\mathrm{Zn}$, apenas a formulação Zapp $\mathrm{Qi}^{\circledR}$ afetou negativamente o cultivar CD $225 \mathrm{RR}$, em comparação ao V Max RR.

Para o fator formulações dos herbicidas, observa-se que o acúmulo de $\mathrm{Cu}$ na parte aérea do cultivar CD $225 \mathrm{RR}$ tratado com Roundup Original ${ }^{\circledR}$ e o da testemunha foram semelhantes entre si, mas inferiores aos das demais formulações. Nota-se também que o acúmulo de $\mathrm{Cu}$ nesse cultivar aumentou com a aplicação dos herbicidas Roundup Ready ${ }^{\circledR}$, Roundup Ultra ${ }^{\circledR}$, Roundup $W^{\circledR}{ }^{\circledR}$ e Zapp Qi ${ }^{\circledR}$. No entanto, para o cultivar V Max RR, a testemunha foi significativamente superior aos tratamentos com Roundup Transorb ${ }^{\circledR} \mathrm{e}$ Roundup $\mathrm{WG}^{\circledR} \mathrm{e}$ igual aos demais tratamentos. No que diz respeito ao $\mathrm{Fe}$, todos os tratamentos não diferenciaram significativamente de sua respectiva testemunha, em ambos os cultivares estudados. Entretanto, no cultivar CD 225 RR, o Roundup Transorb ${ }^{\circledR}$ reduziu o acúmulo de $\mathrm{Fe}$ em relação à formulação Roundup Ready ${ }^{\circledR}$. Para o cultivar V Max RR, os tratamentos com Roundup Original ${ }^{\circledR}$ e Roundup $\mathrm{WG}^{\circledR}$ proporcionaram menor acúmulo que o Zapp QI ${ }^{\circledR}$, sendo a formulação Roundup $\mathrm{WG}^{\circledR}$ também inferior ao Roundup Transorb ${ }^{\circledR} \mathbf{e}$ Roundup Ultra ${ }^{\circledR}$.

Em se tratando do acúmulo de $\mathrm{Mn}$ no cultivar CD $225 \mathrm{RR}$, nenhum tratamento diferiu significativamente da testemunha, porém os tratamentos com Roundup Original ${ }^{\circledR}$ e Roundup Transorb ${ }^{\circledR}$ acumularam menos Mn que o tratamento com Roundup Ultra ${ }^{\circledR}$. Para o cultivar V Max RR, é interessante notar que o tratamento das plantas com o Zapp $\mathrm{Qi}^{\circledR}$ promoveu acréscimo no acúmulo de $\mathrm{Mn}$, porém as outras formulações não exerceram efeito algum no acúmulo desse micronutriente.

Já para o $\mathrm{Zn}$, o acúmulo foi afetado negativamente pelas formulações Roundup Transorb ${ }^{\circledR}$ e Zapp $\mathrm{Qi}^{\circledR}$, em relação à testemunha, no cultivar CD 225 RR. No cultivar V Max RR, todos os tratamentos com as formulações de glyphosate proporcionaram resultados semelhantes ao da testemunha; contudo, o acúmulo de $\mathrm{Zn}$ com a utilização da formulação Zapp Qi ${ }^{\circledR}$ foi superior ao das formulações Roundup Original $^{\circledR}$, Roundup Transorb ${ }^{\circledR}$, Roundup Ultra ${ }^{\circledR}$ e Roundup WG ${ }^{\circledR}$.
Com relação ao acúmulo de $\mathrm{B}$, constatouse que os tratamentos testemunha, Roundup Ready $^{\circledR}$ e Roundup Ultra ${ }^{\circledR}$ foram significativamente superiores aos tratamentos com Roundup Original ${ }^{\circledR}$ e Roundup Transorb ${ }^{\circledR}$ no cultivar CD 225 RR; no entanto, no cultivar V Max RR, as formulações Roundup Ready ${ }^{\circledR} \mathrm{e}$ Zapp $\mathrm{Qi}^{\circledR}$ incrementaram o acúmulo de $\mathrm{B}$ em relação à testemunha.

Comparando os resultados de acúmulo de nutrientes nos cultivares de soja RR com os resultados da análise de nutrientes das caldas de pulverização (Tabela 2), pode-se afirmar que não houve relação direta entre eles, uma vez que a maior ou menor concentração de determinado elemento nas caldas de pulverização não necessariamente condicionou para a mesma tendência no acúmulo deles pelas plantas. Dessa forma, a variação no acúmulo de nutrientes nas plantas de soja RR com a aplicação de diferentes formulações de glyphosate não se deve à presença dos diferentes nutrientes nas caldas de pulverização, e sim a fatores distintos. Nesse aspecto, considerando o acúmulo dos micronutrientes $\mathrm{Mn}$, Zn e B no cultivar V Max RR em relação à testemunha, o seu aumento está relacionado a pequenas variações na produção de matéria seca, principalmente nas plantas tratadas com a formulação Zapp Qi ${ }^{\circledR}$ (Tabela 3). Assim, com maior massa de matéria seca podem-se acumular mais nutrientes. Já para o cultivar CD 225 RR, o incremento de Cu não fica claro com alterações da produção de matéria seca após a aplicação das formulações Roundup Ready $^{\circledR}$ e Roundup Ultra ${ }^{\circledR}$, e outro fator pode estar envolvido no menor acúmulo desse micronutriente no tratamento testemunha. Por outro lado, em termos nutricionais, é pouco provável que o incremento no acúmulo dos micronutrientes em discussão possa favorecer ou não a produção final das plantas tratadas com as formulações de glyphosate, necessitandose estudos adicionais para essa constatação.

Um dos fatores que podem explicar o efeito da redução do acúmulo de nutrientes pelas plantas de soja RR pode estar relacionado, em parte, ao fato de o glyphosate ser um forte quelador de cátions metálicos (Coutinho \& Mazo, 2005), podendo assim se complexar com eles nas plantas, prejudicando a nutrição. $\mathrm{Na}$ formação de complexos metálicos com o 
glyphosate, Barja et al. (2001) relatam que além do grupo fosfonato, que tem habilidade de formar complexos fortes com metais, o herbicida possui outros dois grupos funcionais (amino e carboxilato) que podem se coordenar fortemente com íons metálicos.

Em resumo, para todos os nutrientes avaliados, as formulações Roundup Ready ${ }^{\circledR}$, Roundup Ultra ${ }^{\circledR}$ e Zapp QI ${ }^{\circledR}$ foram as mais seletivas para aplicação nos cultivares CD 225 RR e V Max RR, por não provocarem qualquer comprometimento à nutrição das plantas, quando comparadas aos respectivos tratamentos testemunha sem aplicação. No entanto, Santos et al. (2007b) afirmam que a formulação Roundup Ready ${ }^{\circledR}$ não deve ser aplicada na soja RR em dosagens elevadas, devido ao risco de alterar o teor de alguns nutrientes nas folhas da cultura, entre eles $\mathrm{N}, \mathrm{Ca}, \mathrm{Mg}, \mathrm{Fe}$ e Cu . Por sua vez, as formulações
Roundup Original ${ }^{\circledR}$ e Roundup Transorb ${ }^{\circledR}$ foram as que mais prejudicaram o acúmulo de nutrientes pelas plantas do cultivar CD $225 \mathrm{RR}$, ao passo que a formulação Roundup $\mathrm{WG}^{\circledR}$ se destacou como a menos indicada para aplicação no cultivar V Max RR, em decorrência do efeito negativo provocado no acúmulo dos nutrientes.

No que se refere à produção de matéria seca pelas hastes, folhas e parte aérea (hastes + folhas) dos cultivares de soja RR (Tabela 3), foi constatado o mesmo efeito para o acúmulo de macronutrientes e micronutrientes, sendo o cultivar V Max RR sempre superior ao CD 225 RR quando a diferença entre eles foi significativa. Nesse contexto, apenas a produção de matéria seca pelas folhas no tratamento com Roundup Ready ${ }^{\circledR}$ não apresentou diferença significativa. Segundo Zobiole et al. (2010), efeitos negativos na produção de

Tabela 2 - Concentração de macronutrientes e micronutrientes nas caldas de pulverização das distintas formulações de glyphosate usadas no experimento

\begin{tabular}{|c|c|c|c|c|c|c|c|c|c|c|c|}
\hline \multirow{2}{*}{ Tratamento } & \multicolumn{11}{|c|}{ Macronutrientes e micronutrientes na calda de pulverização $\left(\mathrm{mg} \mathrm{L}^{-1}\right)$} \\
\hline & $\mathrm{N}$ & $\mathrm{P}$ & $\mathrm{K}$ & $\mathrm{Ca}$ & $\mathrm{Mg}$ & $\mathrm{S}$ & $\mathrm{Fe}$ & $\mathrm{Mn}$ & $\mathrm{Zn}$ & $\mathrm{Cu}$ & B \\
\hline Roundup Original $^{(\mathbb{R}}$ & 288,75 & 851,85 & 0,04 & 4,64 & 0,38 & 0,24 & 0,17 & 0,29 & 0,15 & 0,01 & 1,03 \\
\hline Roundup Ready ${ }^{(B)}$ & 261,80 & 784,39 & 0,62 & 4,50 & 0,36 & 0,28 & 0,17 & 0,28 & $<0,01$ & $<0,01$ & 0,74 \\
\hline RoundupTransorb $^{(B)}$ & 272,59 & 846,56 & 0,38 & 4,56 & 0,38 & 0,08 & 0,15 & 0,28 & $<0,01$ & 0,02 & 0,94 \\
\hline Roundup Ultra $^{(R)}$ & 220,84 & 871,69 & 0,60 & 3,86 & 0,32 & 0,96 & 0,16 & 0,28 & 0,05 & 0,19 & 0,35 \\
\hline Roundup WG ${ }^{(R)}$ & 239,47 & 969,58 & 0,04 & 4,66 & 0,40 & 0,44 & 0,31 & 0,29 & 0,34 & 0,04 & 0,52 \\
\hline Zapp Qi ${ }^{(R)}$ & 2,94 & 715,61 & 642,0 & 4,96 & 0,38 & 0,28 & 0,40 & 0,29 & 0,09 & 0,01 & 0,55 \\
\hline
\end{tabular}

Tabela 3 - Produção de matéria seca de hastes, folhas e parte aérea (hastes + folhas) de cultivares de soja aos 15 dias após o tratamento (DAT) com formulações do herbicida glyphosate $(960 \mathrm{~g}$ e. a ha-1 $)$

\begin{tabular}{|c|c|c|c|c|c|c|}
\hline \multirow{3}{*}{ Tratamento } & \multicolumn{6}{|c|}{ Produção de matéria seca (g/planta) } \\
\hline & \multicolumn{2}{|c|}{ Hastes } & \multicolumn{2}{|c|}{ Folhas } & \multicolumn{2}{|c|}{ Hastes + Folhas (Parte Aérea) } \\
\hline & CD $225 \mathrm{RR}$ & V Max RR & CD $225 \mathrm{RR}$ & V Max RR & CD $225 \mathrm{RR}$ & V Max RR \\
\hline Testemunha & $0,29 \mathrm{ab}^{*}$ & $0,40 \mathrm{ab}$ & $0,75 \mathrm{ab}^{*}$ & $0,91 \mathrm{ab}$ & $1,04 \mathrm{ab}^{*}$ & $1,31 \mathrm{ab}$ \\
\hline Roundup Original $^{(\mathbb{R}}$ & $0,22 \mathrm{~cd}^{*}$ & $0,36 \mathrm{~b}$ & $0,53 \mathrm{~cd}^{*}$ & $0,89 \mathrm{ab}$ & $0,74 \mathrm{~cd}^{*}$ & $1,25 \mathrm{~b}$ \\
\hline Roundup Ready ${ }^{(B)}$ & $0,32 \mathrm{a}^{*}$ & $0,38 \mathrm{ab}$ & $0,81 \mathrm{a}$ & $0,94 \mathrm{ab}$ & $1,12 \mathrm{a}^{*}$ & $1,32 \mathrm{ab}$ \\
\hline RoundupTransorb $^{(B)}$ & $0,20 \mathrm{~d}^{*}$ & $0,37 \mathrm{ab}$ & $0,52 \mathrm{~d}^{*}$ & $0,89 \mathrm{~b}$ & $0,71 \mathrm{~d}^{*}$ & $1,26 \mathrm{ab}$ \\
\hline Roundup Ultra $^{(B)}$ & $0,30 \mathrm{ab}^{*}$ & $0,41 \mathrm{ab}$ & $0,74 \mathrm{ab}^{*}$ & $0,96 \mathrm{ab}$ & $1,04 \mathrm{ab}^{*}$ & $1,37 \mathrm{ab}$ \\
\hline Roundup $\mathrm{WG}^{(\mathbb{R}}$ & $0,28 \mathrm{abc}^{*}$ & $0,38 \mathrm{ab}$ & $0,67 \mathrm{bc}^{*}$ & $0,87 \mathrm{~b}$ & $0,94 \mathrm{ab}^{*}$ & $1,25 \mathrm{~b}$ \\
\hline Zapp Qi ${ }^{(B)}$ & 0,25 bcd* & $0,41 \mathrm{a}$ & $0,68 \mathrm{ab}^{*}$ & $1,03 \mathrm{a}$ & $0,92 \mathrm{bc}^{*}$ & $1,45 \mathrm{a}$ \\
\hline DMS & \multicolumn{2}{|c|}{0,06} & \multicolumn{2}{|c|}{0,14} & \multicolumn{2}{|c|}{0,19} \\
\hline $\mathrm{CV}(\%)$ & \multicolumn{2}{|c|}{15,79} & \multicolumn{2}{|c|}{15,49} & \multicolumn{2}{|c|}{14,92} \\
\hline
\end{tabular}

* = médias na linha diferem significativamente pelo teste $\mathrm{F}$ para a variável cultivar $(\mathrm{p}<0,05)$; Médias seguidas pela mesma letra na coluna não diferem significativamente pelo teste $\mathrm{t}(\mathrm{p}<0,05)$. 
matéria seca em plantas de soja RR submetidas à aplicação de glyphosate ocorrem provavelmente devido à diminuição da taxa fotossintética e redução da concentração de nutrientes na parte aérea. Entretanto, nesse trabalho os autores não citam a formulação de glyphosate utilizada nas aplicações.

Quanto ao fator formulações de glyphosate, considerando a produção de matéria seca pelas hastes no cultivar CD $225 \mathrm{RR}$, verificou-se que tratamentos com Roundup Ready ${ }^{\circledR}$, Roundup Ultra $^{\circledR}$, Roundup $\mathrm{WG}^{\circledR}$ e Zapp $\mathrm{Qi}^{\circledR}$ tiveram produção significativamente igual à da testemunha. Os dois primeiros, por sua vez, tiveram produção de matéria seca significativamente maior que a dos tratamentos com Roundup Original ${ }^{\circledR}$ e Roundup Transorb ${ }^{\circledR}$.

Nesse contexto, o tratamento com Roundup Ready ${ }^{\circledR}$ também foi superior ao tratamento com Zapp QI ${ }^{\circledR}$, e o tratamento com Roundup $\mathrm{WG}^{\circledR}$ apresentou maior produção de matéria seca que aquele com Roundup Transorb ${ }^{\circledR}$. Para o cultivar V Max RR, nenhum tratamento diferiu significativamente da testemunha, porém a formulação Zapp Q $I^{\circledR}$ mostrou maior produção de matéria seca pelas hastes que a Roundup Original ${ }^{\circledR}$.

Para a produção de matéria seca pelas folhas, os tratamentos com Roundup Ready ${ }^{\circledR}$, Roundup Ultra ${ }^{\circledR}$ e Zapp Q $i^{\circledR}$ foram estatisticamente iguais ao testemunha para o cultivar CD 225 RR. Nesse caso, a testemunha apresentou maior produção de matéria seca nas folhas que os tratamentos com Roundup Original $^{\circledR}$ e Roundup Transorb ${ }^{\circledR}$. Adicionalmente, além dessas formulações, o tratamento com Roundup Ready ${ }^{\circledR}$ apresentou maior produção de matéria seca que aquele com Roundup $\mathrm{WG}^{\circledR}$. Por outro lado, no cultivar V Max RR, todos os tratamentos não diferiram significativamente da testemunha. Entretanto, a formulação Zapp QI ${ }^{\circledR}$ apresentou maior produção de matéria seca que o Roundup Transorb ${ }^{\circledR}$ e Roundup $\mathrm{WG}^{\circledR}$.

Finalmente, considerando a produção de matéria seca pela parte aérea (hastes + folhas) das plantas de soja RR, verificou-se, para o cultivar CD $225 \mathrm{RR}$, que os tratamentos com Roundup Ready ${ }^{\circledR}$, Roundup Ultra ${ }^{\circledR}$, Roundup $\mathrm{WG}^{\circledR}$ e Zapp $\mathrm{Qi}^{\circledR}$ não diferiram significativamente da testemunha, sendo esta última superior aos tratamentos com a aplicação de Roundup Original ${ }^{\circledR}$ e Roundup Transorb ${ }^{\circledR}$. Todavia, além dessas duas últimas formulações, o tratamento com Roundup Ready ${ }^{\mathbb{R}}$ também apresentou maior produção de matéria seca que o tratamento com Zapp $\mathrm{Qi}^{\circledR}$. Por outro lado, no cultivar V Max RR, assim como verificado para a produção de matéria seca pelas hastes e folhas, não houve diferenças dos tratamentos com herbicidas, se comparado à testemunha. No entanto, o tratamento com Zapp Qi foi superior aos tratamentos com as formulações Roundup Original ${ }^{\circledR}$ e Roundup $\mathrm{WG}^{\circledR}$.

Pode-se ressaltar que as formulações Roundup Ready ${ }^{\circledR}$ e Roundup U1tra ${ }^{\circledR}$ não reduziram a produção de matéria seca tanto das partes da planta (hastes e folhas) quanto da parte aérea (hastes + folhas) do cultivar CD 225 RR, sendo, portanto, indicadas para aplicação em lavouras semeadas com esse cultivar. Já o cultivar V Max RR não apresentou nenhuma restrição à utilização das formulações de glyphosate estudadas, pois os tratamentos com herbicidas não mostraram diferença em relação às respectivas testemunhas, em termos da produção de matéria seca, em todas as avaliações realizadas.

Correia \& Durigan (2007), em estudo com os cultivares CD 214 RR e M-SOY 8008 RR, verificaram que as formulações de glyphosate Roundup Ready ${ }^{\circledR}$, Roundup Transorb ${ }^{\circledR}$, Roundup Original $^{\circledR}$, Roundup $\mathrm{WG}^{\circledR}$, Polaris ${ }^{\circledR}, \mathrm{Gliz}^{\circledR}$, Glifosato Nortox $^{\circledR}$ e Trop $^{\circledR}$ não causaram injúrias às plantas. Esses autores relatam que, apesar da presença de diferentes surfatantes e inertes nas composições dos herbicidas, eles apresentaram comportamentos similares para os cultivares avaliados. No entanto, Santos et al. (2007b) relataram efeito prejudicial da formulação Roundup Transorb ${ }^{\circledR}$, formulado à base do sal de isopropilamina, ao cultivar CD 219 RR, o qual reduziu o número de nódulos radiculares e o rendimento de grãos e promoveu maior efeito negativo sobre a microbiota do solo.

Levando em consideração as formulações de glyphosate quanto à produção de matéria seca pelas plantas dos cultivares de soja RR (CD 225 RR e V Max RR) estudados no experimento, pode-se dizer que elas apresentaram 
efeitos diferenciados, que por sua vez podem ser justificados por vários fatores, entre eles: o sal que compõe a formulação (Reddy \& Zablotowicz, 2003); o surfatante presente na formulação, o qual pode potencializar de forma diferencial a penetração, absorção e translocação do glyphosate pelas plantas (Molin \& Hirase, 2005; Santos et al., 2007a); o efeito em organismos não alvo (Santos et al., 2005, 2006); as disfunções físiológicas provocadas nas plantas, como a redução da lignificação e produção de aminoácidos (Zobiole et al., 2010c), taxa fotossintética e o uso de água pelas plantas (Zobiole et al., 2010c); e o desbalanço nutricional (Zobiole et al., 2009; Serra et al., 2011), além do processo de degradação do herbicida nas plantas, o qual possivelmente resultou na produção de quantidades diferenciadas de metabólitos do glyphosate, entre eles o AMPA (Duke et al., 2003; Reddy et al., 2004).

Todavia, de modo geral, é importante ressaltar, para a soja RR, que fatores como dose aplicada, estádio fenológico de desenvolvimento no momento da aplicação e grupos de maturação dos cultivares podem influenciar em maior ou menor intensidade os efeitos proporcionados pelo glyphosate. Diante disso, mais estudos devem ser realizados visando à obtenção de informações para otimização da tecnologia Roundup Ready na cultura da soja.

Contudo, com base nos resultados apresentados, conclui-se que o acúmulo de nutrientes e produção de matéria seca na parte aérea de plantas de soja é alterado em função da aplicação do glyphosate, mesmo para cultivares RR.

\section{AGRADECIMENTOS}

Os autores agradecem à Fundação de Amparo à Pesquisa do Estado de São Paulo (FAPESP), pela bolsa concedida ao primeiro autor e pelo auxílio financeiro para a condução da presente pesquisa.

\section{LITERATURA CITADA}

BARJA, B. C.; HERSZAGE, J.; AFONSO, M. S. Íons III phosphonate complexes. Polyhedron, v. 20, n. 15-16, p. $1821-1830,2001$
BOTT, S. et al. Glyphosate-induced impairment of plant growth and micronutrient status in glyphosate-resistant soybean (Glycine max L.). Plant Soil, v. 312, n. 1/2, p. $185-194,2008$.

CÉLERES. Relatório de Biotecnologia: Disponível em: $<\mathrm{http}$ // /www.celeres.com.br/1/RelBiotecBrasil_1103.pdf >. Acesso em: 20 mar. 2012.

COMPANHIA NACIONAL DE ABASTECIMENTO CONAB. Acompanhamento de safra brasileira: grãos, décimo levantamento, março 2012/Brasília: 2012. Disponível em: <www.conab.gov.br/conabweb/download/safra/9fraos 8.6.10.pdf>. Acesso em: 20 mar. 2012.

CORREIA, N. M.; DURIGAN, J. C. Seletividade de diferentes herbicidas à base de glyphosate a soja RR. Planta Daninha, v. 25, n. 2, p. 375-379, 2007.

COUTINHO, C. F. B.; MAZO, L. H. Complexos metálicos com o herbicida glyphosate: Revisão. Química Nova, v. 28, n. 6 , p. $1038-1045,2005$

DE MARIA, N. et al. New insights on glyphosate mode of action in nodulant metabolism: role of shikimate accumulation. J. Agric. Food Chem., v. 54, n. 7, p. $2621-2628,2006$

DUKE, S. O. et al. Isoflavone, glyphosate, and aminomethylphosphonic acid levels in seeds of glyphosatetreated, glyphosate-resistant soybean. J. Agric. Food Chem., v. 51, n. 1, p. 340-344, 2003.

INTERNATIONAL SERVICE FOR THE ACQUISITION OF AGRI-BIOTECH APPLICATIONS

AGROBIOTECNOLOGIA - ISAAA. Resumo executivo BRIEF 41 - Situação global das culturas biotecnológicas/ GM comercializadas: 2011. Disponível

em:<www.isaaa.org> Acesso em: 20 mar. 2012.

JOHAL, G. S.; HUBER, D. M. Glyphosate effects on disease and disease resistance in plants. Europ. J. Agron., v. 31, n. 1, p. 144-152, 2009.

KREMER, R. J.; MEANS, N. E. Glyphosate and glyphosate-resistant crop interactions with rhizosphere microorganisms. Europ. J. Agron., v. 31, n. 1, p. 153-161, 2009.

KREMER, R. J.; MEANS, N. E.; KIM, K. S. Glyphosate affects soybean root exudation and rhizosphere microorganisms. Int. J. Environ. Anal. Chem., v. 85, n. 15, p. $1165-1174,2005$.

MOLIN, W. T.; HIRASE, K. Comparison of commercial glyphosate formulations for control of prickly sida, purple nutsedge, morningglory and sicklepod. Weed Biol. Manag., v. 4, n. 3, p. 136-141, 2004.

Planta Daninha, Viçosa-MG, v. 30, n. 2, p. 349-358, 2012 
OLIVEIRA JR., R. S. et al. Influência do glyphosate sobre a nodulação e o crescimento de cultivares de soja resistente ao glyphosate. Planta Daninha, v. 26, n. 4, p. 831-843, 2008

PADGETTE, S. R. et al. New weed control opportunities: development of glyphosate-tolerant soybeans. In: DUKE, S. O. (ed.). Herbicide resistant crops. Boca Raton: CRC, 1995 p. $54-80$.

REDDY, K. N.; RIMANDO, A. M.; DUKE, S. O. Aminomethylphosphonic acid, a metabolite of glyphosate, causes injury in glyphosate-treated, glyphosate resistant soybean. J. Agric. Food Chem., v. 52, n. 16, p. 5139-5143, 2004.

REDDY, K. N.; ZABLOTOWICZ, R. M. Glyphosateresistant soybean response to various salts of glyphosate and glyphosate accumulation in soybean nodules. Weed Sci., v. 51, n. 4, p. $496-502,2003$

SANTOS, J. B. et al. Tolerance of Bradyrhizobium strains to glyphosate formulations. Crop. Protec., v. 24, n. 6 , p. $543-547,2005$.

SANTOS, J. B. et al. Action of two herbicides on the microbial activity of soil cultivated with common bean (Phaseolus vulgaris) in conventional-till and no-till systems. Weed Res., v. 46, n. 1, p. 1-6, 2006

SANTOS, J. B. et al. Efeito de formulações na absorção e translocação do glyphosate em soja transgênica. Planta

Daninha, v. 25, n. 2, p. 381-388, 2007a.

SANTOS, J. B. et al. Avaliação de formulações de glyphosate sobre soja Roundup Ready. Planta Daninha, v. 25, n. 1, p. $165-171,2007 b$.
SERRA, A. P. et al. Influência do glifosato na eficiência nutricional do nitrogênio, manganês, ferro, cobre e zinco em soja resistente ao glifosato. Ci. Rural, v. 41, n. 1, p. 77-84, 2011.

SHANER, D.; BRIDGES, D. Inhibitors of aromatic amino acid biosyntesis (glyphosate). In: HERBICIDE action course. West Lafayette: Purdue University, 2003. p. 514-529.

ZABLOTOWICZ, R. M.; REDDY, K. N. Nitrogenase activity, nitrogen content, and yield responses to glyphosate in glyphosate-resistant soybean. Crop. Protec., v. 26, n. 3, p. 370-376, 2007.

ZOBIOLE, L. H. S. et al. Efeito da compactação do solo e do sulfentrazone sobre a cultura da soja em duas condições de água no solo. Planta Daninha, v. 25, n. 3, p. 537-545, 2007.

ZOBIOLE, L. H. S. et al. Glyphosate reduces shoot concentrations of mineral nutrients in glyphosate-resistant soybeans. Plant Soil, v. 328, n. 1, p. 57-69, 2009.

ZOBIOLE, L. H. S. et al. Effect of glyphosate on symbiotic N2 fixation and nickel concentration in glyphosate-resistant soybeans. Appl. Soil Ecol., v. 44, n. 1, p. 176-180, 2010a.

ZOBIOLE, L. H. S. et al. Glyphosate affects lignin content and amino acid production in glyphosate-resistant soybean. Acta Physiol. Plant., v. 32, n. 5, p. 831-837, $2010 \mathrm{~b}$.

ZOBIOLE, L. H. S. et al. Water use efficiency and photosynthesis of glyphosate-resistant soybean as affected by glyphosate. Pestic. Biochem. Physiol., v. 97, n. 3, p. $182-193,2010 \mathrm{c}$ 